\title{
Irinotecan is inactive as a first-line treatment, but plays an important part in gastric cancer treatment
}

\author{
Hiroya Takiuchi
}

Published online: 1 March 2011

(c) The International Gastric Cancer Association and The Japanese Gastric Cancer Association 2011

Based on the results of the GC0301/TOP002 (S-1 versus irinotecan plus S-1) randomized control study, which were reported in this journal-Gastric Cancer [1]-can we conclude that irinotecan is not important in gastric cancer treatment? Currently, the answer to this question may be "yes", because irinotecan-based regimens did not meet any primary endpoint as a first-line treatment in three randomized control studies, including the GC0301/TOP002 study [1-3]. When these clinical studies were planned, standard treatment in the field of gastric cancer was absent; there was no appropriate, universal control. At that time, the National Comprehensive Cancer Network proposed 5-fluorouracil (FU)-based or cisplatin-based combinations as acceptable standard therapy. 5-FU alone or S-1 (an oral fluoropyrimidine) was standard therapy in Japan, but 5-FU plus cisplatin (CF) was also used frequently. CF was frequently employed in Korea, Japan, many South American countries, and many European countries. Epirubicin plus $\mathrm{CF}$ (ECF) was considered the standard in a few European countries and possibly in Canada. Thus, a 5-FU-based or cisplatin-based combination was an appropriate control in the West and, for that matter, in most of the world. Based on this background, the control arms varied among studies. Three randomized control studies (1 in Europe/the United States, 2 in Japan) were conducted to verify the efficacy of irinotecan-based regimens as a first-line treatment.

A study in Europe and the United States was first reported. In these countries, irinotecan was emphasized as a new active agent for gastric cancer. This study was planned as a phase II/III trial [1]. In the phase II part,

H. Takiuchi $(\bowtie)$

Cancer Chemotherapy Center, Osaka Medical College,

2-7 Daigaku-Cho, Takatsuki, Osaka 569-8686, Japan

e-mail: in2028@ poh.osaka-med.ac.jp irinotecan combined with an infusional 5-FU Arbeitsgemeinschaft Internistische Onkologie (AIO) regimen [irinotecan/5-FU (IF)] was selected over irinotecan combined with cisplatin on the basis of the risk/benefit ratio. At the time, the IF regimen was considered to be the most active irinotecan-based regimen available in the West. In the phase III part, the usefulness of IF in comparison with $\mathrm{CF}$ was examined with respect to the time to progression (TTP) as a primary endpoint. When reviewing the antitumor effects of IF and CF in this study, the response rates (RRs) were 31.8 and $25.8 \%$, respectively. The TTP was 5.0 and 4.2 months, respectively. The median survival time (MST) was 9.0 and 8.7 months, respectively. The toxicity profile of IF was also markedly more favorable than that of CF. However, with respect to the primary endpoint, TTP, the usefulness of IF in comparison with CF could not be demonstrated. In the protocol, it was also impossible to verify the non-inferiority of IF. Based on the results of this study, IF may not become a standard regimen for first-line treatment; the first study was unsuccessful in the West.

Combination therapy with irinotecan and cisplatin (IC) was initially investigated as a first-line treatment in the JCOG9912 study, which was conducted by the Japan Clinical Oncology Group (JCOG); in this study, the usefulness of combination therapy with IC in comparison with the continuous intravenous infusion of 5-FU (5-FUci) was examined [2]. The RR and time to treatment failure (TTF) were significantly more favorable with IC. However, with respect to the primary endpoint, overall survival (OS), the usefulness of combination therapy with IC in comparison with 5-FUci was not demonstrated to be statistically significant $(p=0.055)$. Clinical studies not only in Europe and the United States but also in Japan failed to propose combination therapy with IC as a standard treatment. When reviewing the survival curve for this combination therapy 
in the JCOG study, the curve within 1 year was markedly more favorable than that for 5-FUci. However, the curve crossed with that for S-1, which was shown to be as effective as 5-FUci, and finally overlapped the survival curve for 5-FUci. On the other hand, the survival curve for S-1 was superior to that for 5-FUci, showing a favorable survival curve. In addition, with respect to prolonged survival, this curve exceeded that for combination therapy with IC. This aspect is important for evaluating the role of irinotecan for gastric cancer treatment.

Based on the results of the JCOG9912 study, singleagent therapy with S-1 has been introduced as a standard regimen in Japan. In a randomized control study reported in this journal, the efficacy of combination therapy with irinotecan and S-1 (IRIS) was investigated [1]. The response rates for S-1 alone and IRIS were 26.9 and $41.5 \%$, respectively, showing a significant difference $(p=0.035)$. Concerning the toxicity, the incidence of Grade 3 or higher adverse reactions after IRIS administration was slightly higher than that after the administration of S-1 alone. However, the adverse reactions were tolerable. The TTF for S-1 alone and IRIS was 3.6 and 4.5 months, respectively; there was no significant difference $(p=0.157)$. With respect to the primary endpoint in this study, OS, the MST on the predetermined cut-off date in the IRIS and S-1 monotherapy groups was 12.8 and 10.5 months, respectively (hazard ratio [HR]: $0.856 ; p=0.223$ ), showing no significant difference. The 1-year survival rates were 52.0 and $44.9 \%$, respectively, showing an approximately $7 \%$ difference. However, the 2-year survival rates were 18.0 and $19.5 \%$, respectively; there was no significant difference between the two arms. After 18 months of treatment, the survival curves for the two therapies overlapped. The results of this study were consistent with those of the JCOG9912 study. On the other hand, the SPIRITS study indicated that the OS in patients treated with cisplatin plus S-1 was significantly longer than that in those treated with S-1 alone [4]. Differences between cisplatin and irinotecan as first-line treatment must be reviewed.

Second-line and subsequent treatment has been important for examining the prolongation of survival in patients with gastric cancer in recently reported clinical studies. In Japan, second-line and subsequent treatment is fully covered by health insurance, differing from the health insurance coverage in Europe and the United States. Recently, second-line treatment has been performed in approximately $80 \%$ of the subjects of clinical studies in Japan [1, 3, 4]. In the JCOG9205 study, which was conducted prior to the JCOG9912 study, 5-FUci was also employed as a control arm [5]. This regimen, which was common between the two studies, prolonged the MST by 3.7 months (range 7.1-10.8 months) in the JCOG9912 study. This was possibly because the proportion of patients in whom second- line treatment was introduced had increased [6]. In addition, another factor was an increase in the number of agents that may be active for gastric cancer treatment. Currently, four agents and agent classes, fluoropyrimidine, cisplatin, taxane, and irinotecan, are considered to be effective for the treatment of gastric cancer. Of these, cisplatin is appropriate for combination therapy in first-line treatment, for the following reasons: monotherapy with cisplatin is not effective, and its toxicity is greater than moderate. On the other hand, taxane and irinotecan are commonly used as single agents for second-line and subsequent treatment in Japan [3, 4]. The results of the AIO study comparing the best supportive care with irinotecan alone in second-line treatment, which was reported at the American Society of Clinical Oncology (ASCO) 2009 annual meeting, suggested the usefulness of irinotecan in second-line treatment for gastric cancer [7]. We must review why negative results were obtained in 3 studies in which irinotecan was verified for first-line treatment. We should remember the fact that an irinotecan-based regimen did not show any favorable survival curve with respect to long-term survival. This suggests that, when employing irinotecan as a first-line agent, a second-line or subsequent treatment option is lost. This is the most important difference between cisplatin and irinotecan. The potential benefit of irinotecan-based regimens has been further explored in the past few years, especially with the availability of new targeted agents. To apply irinotecan as a first-line agent in the future, combination therapy with new targeted agents should be performed.

\section{References}

1. Narahara $H$, Iishi $H$, Imamura $H$, Tuburaya $A$, Chin $K$, Imamoto $H$, et al. Randomized phase III study comparing the efficacy and safety of irinotecan plus S-1 with S-1 alone as first-line treatment for advanced gastric cancer (study GC0301/TOP-002). Gastric Cancer, doi:10.1007/s10120-011-0009-5.

2. Dank M, Zaluski J, Barone C, Valvere V, Yalcin S, Peschel C, et al. Randomized phase III study comparing irinotecan combined with 5-fluorouracil and folinic acid to cisplatin combined with 5-fluorouracil in chemotherapy naive patients with advanced adenocarcinoma of the stomach or esophagogastric junction. Ann Oncol. 2008;19:1450-7.

3. Boku N, Yamamoto S, Fukuda H, Shirao K, Doi T, Sawaki A, et al. Fluorouracil versus combination of irinotecan plus cisplatin versus S-1 in metastatic gastric cancer: a randomized phase 3 study. Lancet Oncol. 2009;10:1063-9.

4. Koizumi W, Narahara H, Hara T, Takagane A, Akiya T, Takagi M, et al. Randomized phase III study of S-1 alone versus S-1 + cisplatin in the treatment for advanced gastric cancer (The SPIRITS trial) SPIRITS: S-1 plus cisplatin vs S-1 in RCT in the treatment for stomach cancer. Lancet Oncol. 2008;9:215-21.

5. Ohtsu A, Shimada Y, Shirao K, Boku N, Hyodo I, Saito H, et al. Randomized phase III trial of fluorouracil alone versus fluorouracil 
plus cisplatin versus uracil and tegafur plus mitomycin in patients with advanced gastric cancer: JCOG study 9205. J Clin Oncol. 2003;21:54-9.

6. Takashima A, Boku N, Kato K, Mizusawa J, Nakamura K, Fukuda $\mathrm{H}$, et al. Survival prolongation after treatment failure in patients with advanced gastric cancer (AGC): results from combined analysis of JCOG9205 and JCOG9912 [abstract no. 4061]. J Clin Oncol. 2010;28(Suppl 15S):4061.
7. Thuss-Patience PC, Kretzschmar A, Deist T, Hinke A, Bichev D, Lebedinzew B, et al. Irinotecan versus best supportive care (BSC) as second-line therapy in gastric cancer: a randomized phase III study of the Arbeitsgemeinschaft Internistische Onkologie (AIO) [abstract no. 4540]. J Clin Oncol. 2009;27(Suppl 15S):4540. 